\title{
Validade Convergente entre Processos Cognitivos do Zulliger com Idade e Escolaridade
}

\author{
Jucelaine Bier Di Domenico Grazziotin \\ Silvana Alba Scortegagna
}

\section{RESUMO}

Este estudo buscou evidências de validade convergente entre indicadores do processamento cognitivo do teste de Zulliger com a idade e a escolaridade. Participaram 64 adultos não pacientes, entre 18 e 59 anos de idade $(M=30,14, D P=10,33)$, prestadores de serviços comerciais. Utilizaram-se como instrumentos um questionário sociodemográfico e o teste de Zulliger no Sistema Compreensivo. Na comparação com a amostra normativa brasileira, houve aumento significativo nas variáveis do Zulliger que indicam capacidade de análise e síntese (DQ+, $\mathrm{W}, \mathrm{XA} \%$ ), e rebaixamento significativo de indicadores que mostram percepção inadequada da realidade $(\mathrm{X}-\%)$. A associação do Zulliger com as variáveis externas, a correlação significativa (W:M) mostrou menos tendência à liderança e ambição intelectual em adultos mais jovens. A escolaridade demonstrou associação significativa com recursos intelectuais e iniciativa na tomada de decisões (M, Ma:Mp). A convergência do Zulliger com idade e escolaridade potencializa o uso do instrumento e enriquece a compreensão de aspectos cognitivos de adultos.

Palavras-chave: validade do teste; técnicas projetivas; traços de personalidade; psicologia organizacional.

\section{ABSTRACT \\ Convergent Validity between Zulliger Cognitive Processes with Age and Schooling}

This study sought evidence of convergent validity between indicators of cognitive processing of the Zulliger test with age and schooling. There were Sixty-four adults not patients, aged between 18 and 59 years $(M=30,14, D P=10,33)$, service providers in commercial. The instruments used were a sociodemographic questionnaire and Zulliger in the Comprehensive System. In comparison with the Brazilian normative sample there was a significant increase in Zulliger variables that indicate analysis and synthesis capacity (DQ +, W, XA\%), and significant reduction of indicators that show an inadequate perception of reality $(\mathrm{X}-\%)$. In the association of the Zulliger with the external variables, the significant correlation (W: M) showed a less tendency to leadership and intellectual ambition capacity in younger adults. Schooling showed a significant association with intellectual resources and decision making initiative (M, Ma: Mp). The results evidenced the convergent validity of the Zulliger with age and schooling, enriching the understanding of the cognitive aspects of adults.

Keywords: test validity; projective techniques; personality traits; organizational psychology.

Trabalhadores comerciais empreendedores geralmente ascendem à carreira, deixam de exercer funções com foco na tarefa e execução e migram para funções de comando, supervisão e organização. Este fenômeno resulta, em parte, do equilíbrio nos processos cognitivos, entre a capacidade de raciocínio e o acúmulo de conhecimento e experiências relevantes (Agarwal et. al., 2009; Salthouse, 2012).

\section{Sobre os autores}

J. B. D. D. G.

http://orcid.org/0000-0002-

0147-7498

Universidade de Passo Fundo Passo Fundo, RS

jucelainegraz@terra.com.br

S. A. S.

http://orcid.org/0000-0002-

5100-6459

Universidade de Passo Fundo Passo Fundo, RS

silvanalba@upf.br

\section{Direitos Autorais}

Este é um artigo de acesso aberto e pode ser reproduzido livremente, distribuído, transmitido ou modificado, por qualquer pessoa desde que usado sem fins comerciais. $O$ trabalho é disponibilizado sob a licença Creative Commons CC-BY-NC.

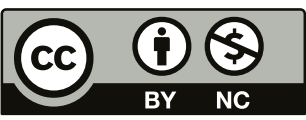




\section{M." INTERACÃO EM PSICOLOGIA}

Os processos cognitivos podem sofrer variações ao longo da vida, algumas habilidades podem melhorar, outras podem permanecer estáveis ou diminuírem (Argimon et. al., 2014; Salthouse, 2012;). Pode ocorrer declínio da velocidade de processamento da informação (inteligência fluida), as habilidades cristalizadas geralmente mostram pouca ou nenhuma diferença (Salthouse, 2012; Hartshorne \& Germine, 2015).

Adultos jovens e de meia idade encontram-se no auge das capacidades cognitivas, produtivas (Agarwal et al., 2009; Salthouse, 2012), geralmente possuem baixa probabilidade de distorção da percepção (Franco \& Villemor-Amaral, 2012; Meyer et. al., 2015). Avaliar precocemente as mudanças cognitivas que possam ocorrer durante a fase adulta é tarefa complexa, mas necessária no sentido de subsidiar meios para intervir preventivamente contra problemas cognitivos (Cabeza et al., 2018; Salthouse, 2012, Meléndez et. al., 2013). Condições de saúde, participação em atividades ocupacionais e nível de escolaridade atuam como um fator de proteção e potencialização cognitiva em todas as idades (Meléndez et al., 2013; Zimmermann et. al. 2014).

Profissionais com maior nível de escolaridade na área em que atuam frequentemente são mais bem-sucedidos no mundo do trabalho. Estudos iniciais mostraram associação entre o nível elevado de escolaridade de adultos com maior produtividade, respostas de movimento humano e diminuição das respostas determinadas apenas pela forma no método de Rorschach (Ames, et. al., 1973). Mais recentemente, o aumento da educação esteve associado significativamente com variáveis do método de Rorschach de síntese cognitiva, produtividade e com indicadoras de recursos de enfrentamento (Meyer et. al., 2011; Meyer et al., 2015, Pires, 2000). Estes resultados convergentes denotam associação entre o nível educacional e os escores do método de Rorschach, a necessidade de estudos que considerem a avaliação cognitiva com o uso de testes psicológicos e os efeitos de variáveis demográficas.

Meyer et al. (2015) examinaram a associação de gênero, etnia, idade e escolaridade em escores de Rorschach com três tipos de amostras: não clínica (18 a 86 anos, $n=640$ ); clínica de adultos (18 a 77 anos, $n=249$ ) e de crianças e jovens ( 5 a 16 anos, $n=241$ ). Entre os resultados, na amostra adultos não clínica, a idade esteve associada positivamente com Sombreado Textura (T) e resposta personalizada (PER). As variáveis Movimento Humano (M), Soma de conteúdos humanos (Sum $H)$, respostas globais (W\%) apresentaram-se aumentadas quando associadas a mais anos de educação enquanto que a porcentagem de forma Pura (F\%) e respostas de detalhe inusual (Dd\%) mostraram-se diminuídas quando associadas aos anos de educação. Na amostra clínica de crianças e jovens, a idade foi associada com a percepção mais convencional e percepção de pensamento menos distorcido.
Jucelaine Bier Di Domenico Grazziotin e Silvana Alba Scortegagna
Um dos instrumentos mais econômicos em termos de tempo que tem suas raízes no método de Rorschach é o Teste de Zulliger. O Rorschach é um instrumento amplamente pesquisado e com muitos avanços em sua administração, codificação e interpretação. Embora estudos indiquem pouca concordância entre os dois instrumentos (Villemor-Amaral et. al., 2016), a fundamentação teórica, aplicação e interpretação guardam semelhanças e os avanços no estudo do Rorschach auxiliam na interpretação e na busca de aperfeiçoamento do Zulliger (Gonçalves et. al., 2019; Villemor Amaral \& Primi, 2012). O Zulliger é composto por três estímulos de manchas de tinta semiestruturados. Inicialmente, o entrevistado explicita com o que a mancha lhe parece e, posteriormente, explica a base da resposta. Isso fornece respostas comportamentais a uma tarefa complexa de solução de problemas, por meio de componentes visuais, cognitivos e perceptivos que envolvem o processamento implícito de organizar as semelhanças perceptuais incompletas e imperfeitas de maneira que Ihes faça sentido (Viglione et al., 2015; Villemor Amaral \& Cardoso, 2018).

Em contextos empresariais, a escolha por métodos de avaliação que possuem tais características é particularmente importante. Tal importância se deve, em procedimentos de seleção profissional e para ascensão na carreira, a os examinandos poderem comportar-se melhor do que são e responder aos testes de autorrelato de maneira mais condizente com as expectativas do empregador (Widhiarso \& Himam, 2015), o que é difícil de ocorrer quando respondem aos métodos de manchas de tinta ou projetivos. Muito embora as características autorreferidas respondam mais comumente às avaliações da personalidade (Viglione et al., 2015) as manchas de tinta fornecem um método alternativo e complementar valioso, pois tem potencial de revelar especificidades do mundo interno e podem ser úteis para integrar resultados autorrelatados (Evans \& Finn, 2017; Meyer, 2017).

Avaliações psicológicas estão fortemente atreladas à competência técnica, à ética do profissional e à validade dos instrumentos (Meyer et al., 2015; Primi et. al., 2012). De modo geral, este tripé sustenta avaliações de qualidade que podem beneficiar pessoas e/ou populações em contextos diversos. Em se tratando dos instrumentos psicológicos, acumular evidências de validade que certifiquem sua relevância nos usos propostos e nas interpretações geradas é um meio de garantir que a Psicologia possa exercer seu compromisso ético e social (Conselho Federal de Psicologia - CFP, 2018). Estudos de evidências de validade do Teste de Zulliger e que abordam aspectos cognitivos foram conduzidos no âmbito empresarial e clínico (Ferreira \& Villemor-Amaral, 2005; Franco \& Villemor-Amaral, 2012; Grazziotin \& Scortegagna, 2016a, 2018). No contexto empresarial, Ferreira e Villemor-Amaral (2005), buscaram evidências de validade para o Teste de Zulliger por meio da correlação com um questionário de Avaliação de 


\section{H*' INTERACÃO EM LTPSICOLOGIA}

Jucelaine Bier Di Domenico Grazziotin e Silvana Alba Scortegagna
Desempenho. A amostra constitui-se de 86 profissionais com idades entre 22 a 43 anos. Entre os resultados, a proporção de movimento humano ativo e passivo Ma:Mp correlacionou-se com solução de problemas e a soma de movimento humano (Sum M) com tomadas de decisões oportunas. Grazziotin e Scortegagna (2016a) investigaram a validade do Teste de Zulliger, focalizando variáveis da mediação, em amostra gaúcha de 40 trabalhadores comerciais, não pacientes, com idades entre 18 e 43 anos. Comparando-se com os dados normativos brasileiros, as análises demonstraram diferenças estatisticamente significativas no aumento das variáveis que se referem à percepção adequada da realidade ( $X A \%, W D A \%$, $X+\%)$, no rebaixamento das variáveis que indicam percepção distorcida da realidade ( $X$-\% e $S$-) no grupo gaúcho e evidenciaram a validade do instrumento para o contexto pesquisado. O estudo incrementou a relevância do Zulliger ao demonstrar especificidades do comportamento humano, notadamente trabalhadores do comercio gaúcho.

No contexto clínico, Franco e Villemor-Amaral (2012) buscaram a validade incremental de duas técnicas projetivas, Zulliger e Teste das Pirâmides de Pfister (TPC), com amostra de 20 dependentes químicos, entre 21 a 45 anos de idade. Os resultados indicaram dificuldades entre os integrantes da amostra para respeitar as regras sociais, observadas pela diminuição na frequência de respostas populares $(P)$. Os protocolos dos pacientes que não reagiram bem ao tratamento de desintoxicação das drogas evidenciaram sinais de personalidade mais desconectada da realidade, demonstrado pelo aumento de X-\%.

Recentemente, Grazziotin e Scortegagna (2018) buscaram a convergência dos indicadores do relacionamento interpessoal e da tríade cognitiva do Zulliger com o Inventário de Habilidades Sociais para Idosos (IHSI Del-Prette). 0 estudo foi conduzido com amostra de 78 idosos, com idades entre 60 e 96 anos. Os achados evidenciaram a validade convergente do Zulliger com IHSI Del-Prette, houve associações significativas das variáveis do relacionamento interpessoal e da tríade cognitiva do Zulliger ( $R, D, X A \%, S-, X+\%)$ com o escore geral G e os fatores F4, F2, F3 e F1 do IHSI Del-Prette.

0 teste de Zulliger mostra potencial para responder às demandas de avaliação que incluem aspectos cognitivos. No entanto, pode-se observar que são quase inexistentes estudos com a sua utilização que abordem os aspectos cognitivos em não pacientes e, que se tenha conhecimento, são inexistentes os estudos que verifiquem a relação desta técnica projetiva com variáveis como a idade e a escolaridade. Em revisão de literatura, Grazziotin e Scortegagna (2016b) encontraram somente 12 artigos com o uso do instrumento, no período de 2004 a 2014, apenas três com foco no contexto empresarial e que não abordaram questões de idade, escolaridade e cognição. Em concordância com Cardoso, Gomes, Pacheco \& Viana
(2018) e Villemor Amaral \& Cardoso (2018) há necessidade de atualizações e incremento de estudos com as qualidades psicométricas do instrumento.

Nesta perspectiva, Villemor-Amaral \& Cardoso (2018) acrescentam que para uma boa análise do Teste de Zulliger é desejável que a pessoa examinada forneça um número suficiente de respostas. Protocolos com menos de seis respostas podem prejudicar a confiabilidade das interpretações. Contribuições do Rorschach Performance Assessment System (R-PAS, Meyer, 2017; Viglione et al.,2015), e estudos incipientes com o Teste de Zulliger (Gonçalves et al., 2019; Villemor-Amaral et al., 2016) sugerem controlar o número de respostas para evitar a disparidade de protocolos muito curtos ou extensos demais e aconselham a otimizar o número de respostas de modo que os respondentes deem de três a cinco respostas por cartão, totalizando de nove a quinze respostas em um protocolo.

Como se pode observar, são muitas as justificativas que impulsionaram o desenvolvimento deste estudo. Resumidamente, destacam-se quatro delas: i) com o avanço do curso de vida pode ocorrer declínio na velocidade de processamento de informação, memória de trabalho e organização perceptual (Salthouse, 2012); ii) adultos mais velhos saudáveis, com alto nível de escolaridade, avaliados pelo Teste de Zulliger, apresentam cognição preservada (Aguilar \& Graso, 2015); iii) a produtividade no método de Rorschach deve ser associada com educação, inteligência e adaptação (Meyer et al., 2011); iv) a educação parece ser a variável de associação mais consistente com os escores do método de Rorschach em adultos (Meyer et al., 2015). Estes achados têm potencial de impactar diferentes aspectos da sociedade contemporânea, especialmente o mundo do trabalho. Portanto, constitui-se objetivo principal deste estudo buscar evidências de validade convergente entre indicadores do processamento cognitivo do Teste de Zulliger com a idade e a escolaridade.

Partindo do pressuposto de que se as variáveis $R, P, M$, $M a: M p, D Q+$ são representativas da produtividade, motivação, empatia, de iniciativa e capacidade cognitiva, se as variáveis $X A \%$ e $X-\%$, indicam a capacidade de percepção e adequação social (Villemor-Amaral \& Primi, 2012; Exner, 2003), tem-se como hipóteses: $(\mathrm{H} 1)$ em indivíduos com maior escolaridade haverá aumento do número de respostas $(R)$ e de respostas populares $(P)$, de movimento humano $(M)$, da proporção do movimento humano ativo em relação ao passivo (Ma:Mp), respostas sintetizadas $(D Q+)$, respostas de forma adequada com mínimo de distorções (XA\%) e diminuição de respostas de forma distorcida $(X-\%)$. Se as variáveis $W, W: M, M, M a: M p$ são indicativas da capacidade de planejamento, organização perceptual e aspiração intelectual, iniciativa na tomada de decisões (Villemor-Amaral \& Primi, 2012 Exner, 2003) pode-se hipotetizar que: $(\mathrm{H} 2)$ em indivíduos mais velhos haverá 


\section{H* INTERACÃO EM L PSICOLOGIA}

aumento das respostas globais $(W)$, da proporção de respostas globais em relação ao movimento humano (W:M), e diminuição do movimento humano $(M)$, e de movimento humano ativo em relação ao passivo (Ma:Mp)

\section{MÉTODO}

\section{PARTICIPANTES}

A amostra intencional constitui-se de 64 adultos não pacientes, sem diagnóstico de doença mental, tratamento psiquiátrico ou psicológico, com idades entre 18 a 59 anos $(M=30,14, D P=10,33)$, de 18 a 29 anos $(50 \%)$ e 30 a 59 anos (50\%), pareados em gênero, com nível socioeconômico pertencentes às classes $\mathrm{C} 1$ (62,55\%) e C2 (29,70\%), escolaridade entre 7 a 13 anos de estudo $(M=9,5 D P=2,74)$, de 7 a 10 anos de estudo (45,31\%) e 11 a 13 anos de estudo (54,68\%), atendentes de lojas de supermercados e prestadores de serviços nos estabelecimentos. Os que se concentravam nas funções de operadores de caixa e atendentes de loja $(71,90 \%)$ integravam as equipes de funcionários dos supermercados e os que ocupavam funções de vendedores, promotores, fornecedores e entregadores de mercadorias $(28,10 \%)$ eram prestadores de serviço e representavam outras atividades do comércio como lojas, distribuidores de bebidas e de alimentos. Todos trabaIhavam diretamente com atendimento a clientes, sendo que $35 \%$ dos participantes com idades entre 25 a 59 anos ocupavam cargos de supervisão do setor, em diversas lojas, localizados em diferentes zonas geográficas do Município.

Considerou-se como critérios de inclusão: a) vendedores, fornecedores, promotores de venda, operadores de caixa, atendentes de loja com no mínimo 90 dias na função, e b) realizado avaliações psicológicas e médicas para ingresso na empresa e considerados aptos para trabalhar de acordo com o Programa de Controle Médico de Saúde Ocupacional-PCMSO (Ministério do Trabalho, 2018).

Como critérios de exclusão foram considerados, os que exibissem: (a) histórico de tratamento psiquiátrico e/ou de situações estressoras decorrentes do diagnóstico de doença ou de incapacidade física, nos últimos seis meses; (b) histórico de alguma perda importante que acarretou sofrimento, nos últimos seis meses; (c) déficit auditivo e visual que interferisse na execução dos testes.

\section{INSTRUMENTOS}

Questionário Sociodemográfico: com o objetivo de verificar os critérios de inclusão/exclusão da amostra. Protocolo contendo registros de endereço, idade, gênero, escolaridade, classe socioeconômica, salário, profissão ou ocupação, avaliações psicológicas e médicas (PCMSO) e condições de
Jucelaine Bier Di Domenico Grazziotin e Silvana Alba Scortegagna saúde (tratamento psiquiátrico, situações estressoras, diagnóstico de doença mental ou de severa incapacidade física; perdas importantes, problemas de audição e de visão).

Teste de Zulliger no Sistema Compreensivo (ZSC; Villemor-Amaral \& Primi, 2012), possibilita a interpretação da dinâmica da personalidade e dos processos cognitivos. Composto por um jogo de três cartões com manchas de tinta. Os estudos de fidedignidade do teste, por meio do método teste-reteste e confiabilidade entre avaliadores, indicaram correlação acima de $r=0,60$ entre primeira e a segunda aplicação (intervalo de 5 meses), demonstrando uma associação alta e valor acima de $\boldsymbol{\alpha}=0,70$ para concordância entre juízes. Para atender ao objetivo deste estudo, selecionaram-se variáveis vinculadas às capacidades cognitivas e produtivas: respostas globais $(W)$, respostas de movimento humano $(M)$, proporção de respostas globais e de movimento humano $(W: M)$, respostas sintetizadas $(D Q+)$, de forma apropriada estendida $(X A \%)$, de forma distorcida $(X-\%)$, respostas populares $(P)$, proporção de movimento humano ativo e passivo $(M a-M p)$. Incluiu-se o número de respostas $(R)$ por também representar recursos cognitivos.

\section{PROCEDIMENTOS}

Após a aprovação do estudo pelo Comitê de Ética em Pesquisa de uma Universidade localizada no Rio Grande do Sul, protocolo número $042 / 2013$, iniciou-se a coleta de dados. Os participantes foram indicados pelos gerentes dos supermercados com a ficha profissional e de saúde. Os que aceitaram participar da pesquisa assinaram o Termo de Consentimento Livre e Esclarecido e responderam aos instrumentos individualmente, nas dependências das empresas, em um tempo estimado de 50 minutos. Inicialmente foi administrado o Questionário Sociodemográfico e, na sequência, o teste de Zulliger. A aplicação dos instrumentos foi realizada pela autora principal deste estudo (80\%) e por uma aluna do quinto ano do Curso de Psicologia (20\%), devidamente treinada e supervisionada.

Para maior confiabilidade dos dados do teste de Zulliger, conduziu-se um estudo de fidedignidade entre avaliadores. Foram sorteados $25 \%$ dos protocolos, considerando a proporcionalidade entre diferentes aplicadores, e encaminhados para recodificação de um juiz independente, procedendo, a posteriori, à análise do coeficiente Kappa. Obteve-se valores de 0,82 e acima deste para todas as variáveis do presente estudo: W $(1,00), M(0,95), M a(0,92), M p(0,82), F Q+(0,99)$, FQo $(0,92)$ FQu $(0,88)$ FQ- $(0,86)$. Tal coeficiente de precisão revela um índice ótimo de confiabilidade (Landis \& Koch, 1977). 


\section{H MTERAC̄OOEM ET PSICOLOGIA}

Jucelaine Bier Di Domenico Grazziotin e Silvana Alba Scortegagna

\section{ANÁLISE DOS DADOS}

Primeiramente, para verificar as características da amostra, foi realizada uma comparação das variáveis da tríade cognitiva do Zulliger com os parâmetros normativos nacionais de não pacientes (Franco et. al., 2012), utilizando-se o método estatístico t de Student e a magnitude das diferenças obtidas através do $d$ de Cohen. A seguir, para verificar a ocorrência de associação entre as variáveis da tríade cognitiva do Zulliger com a idade e a escolaridade, empregou-se a correlação de Pearson $(p \leq 0,05)$.

\section{RESULTADOS}

Para dar melhor visibilidade aos achados, os dados obtidos serão apresentados em forma de tabelas com os indicadores das variáveis pesquisadas e, na sequência, prossegue-se com a discussão dos resultados. A Tabela 1 apresenta as médias e desvio padrão das variáveis da presente amostra, da amostra normativa e os valores do método estatístico t de Student, dod de Cohen e de $p$.

Tabela 1. Comparação entre as amostras nas variáveis cognitivas do Zulliger

\begin{tabular}{cccccccc}
\hline & \multicolumn{3}{c}{$\begin{array}{c}\text { Amostra do Estudo } \\
(\mathrm{N}=64)\end{array}$} & \multicolumn{4}{c}{$\begin{array}{c}\text { Amostra Normativa } \\
(\mathrm{N}=220)\end{array}$} \\
\hline Variáveis & $\mathrm{M}$ & $\mathrm{DP}$ & $\mathrm{M}$ & $\mathrm{DP}$ & $\mathrm{t}$ & $\mathrm{d}$ & $\mathrm{p}$ \\
\hline $\mathrm{R}$ & 8,20 & 2,13 & 8,2 & 3,1 & 0,120 & 0,03 & 0,99 \\
W & $1,64 *$ & 1,30 & 1,3 & 1,1 & 2.086 & $0,30 *$ & 0,04 \\
W : M & 0,65 & 1,55 & 0,4 & 1,5 & 1.164 & 0,17 & 0,20 \\
M & 1,00 & 0,87 & 0,9 & 1,1 & 0,669 & 0,10 & 0,37 \\
DQ+ & $2,03 *$ & 1,12 & 1,4 & 1,4 & 3.304 & $0,47 *$ & 0,01 \\
XA\% & $83,66 *$ & 11,16 & 73 & 21 & 3.901 & $0,55 *$ & 0,01 \\
X-\% & $15,32 *$ & 11,20 & 21 & 15,9 & -2.670 & $0,38 *$ & 0,01 \\
P & 1,56 & 0,94 & 1,4 & 1,1 & 1.056 & 0,15 & 0,17 \\
Ma - Mp & 0,48 & 0,70 & 0,4 & 0.9 & 0,655 & 0,09 & 0,74 \\
\hline
\end{tabular}

Nota. Legenda das variáveis do Zulliger: Numero de respostas (R). respostas globais $(W)$, proporção de globais e movimento humano $(W: M)$, movimento humano $(M)$, sintetizadas $(D Q+)$, respostas de forma apropriada estendida $(X A \%)$, de forma distorcida $(X-\%)$, populares $(P)$, movimento humano ativo menos passivo (Ma-Mp),

A Tabela 1 demonstra que quatro indicadores das variáveis pesquisadas, da tríade cognitiva, foram compatíveis aos apresentados pela amostra normativa brasileira de não pacientes (Villemor-Amaral \& Primi, 2012). Os participantes deste estudo emitiram de duas a cinco respostas por cartão, obtendo-se protocolos com um total de no mínimo seis e no máximo 14 respostas, denotaram níveis adequados de produtividade e motivação para a tarefa $(\mathrm{R})$, criatividade, raciocínio abstrato e empatia (M), tomada de decisão (Ma-Mp), e adequação social $(P)$. Destaca-se elevada capacidade de análise, síntese e abstração, demonstrado pelo aumento significativo das respostas sintetizadas $(\mathrm{DQ}+)$ e globais $(\mathrm{W})$, e de um raciocínio lógico e adequado ao ambiente, denotado pelo aumento significativo de respostas de forma apropriada (XA\%) e da diminuição significativa das respostas de forma distorcida (X-\%).

A Tabela 2 apresenta os resultados das associações entre as variáveis da cognição do teste de Zulliger com a idade e a escolaridade, por meio da correlação de Pearson.

Tabela 2. Associação entre idade e escolaridade com as variáveis do Zulliger

\begin{tabular}{|c|c|c|c|c|}
\hline \multirow{2}{*}{$\begin{array}{c}\begin{array}{c}\text { Empresa } \\
(\mathrm{n}=64)\end{array} \\
\text { Variáveis }\end{array}$} & \multicolumn{2}{|c|}{ Idade } & \multicolumn{2}{|c|}{ Escolaridade } \\
\hline & $\mathbf{r}$ & p & $r$ & $\mathbf{p}$ \\
\hline $\mathrm{R}$ & $-0,118$ & 0,353 & 0,142 & 0,262 \\
\hline W & 0,133 & 0,293 & $-0,009$ & 0,942 \\
\hline $\mathrm{W}: \mathrm{M}$ & 0,290 & 0,020 * & $-0,176$ & 0,165 \\
\hline$M$ & $-0,312$ & 0,012 * & 0,308 & $0,013 *$ \\
\hline $\mathrm{DQ}+$ & $-0,051$ & 0,687 & 0,153 & 0,229 \\
\hline$X A \%$ & 0,095 & 0,455 & 0,223 & 0,076 \\
\hline$X-\%$ & $-0,078$ & 0,541 & $-0,234$ & 0,063 \\
\hline$P$ & $-0,081$ & 0,527 & 0,346 & 0,005 * \\
\hline $\mathrm{Ma}-\mathrm{Mp}$ & $-0,287$ & 0,021 * & 0,348 & $0,005 *$ \\
\hline Idade e & $r$ & $p$ & & \\
\hline Escolaridade & $-0,487$ & 0,001 & & \\
\hline
\end{tabular}

Nota. Legenda das variáveis do Zulliger: Numero de respostas (R).respostas globais $(W)$, proporção de globais e movimento humano (W: $M)$, movimento humano (M), sintetizadas (DQ+), respostas de forma apropriada estendida (XA\%), de forma distorcida (X-\%), populares $(\mathrm{P})$, movimento humano ativo menos passivo (Ma-Mp).

A Tabela 2 apresenta correlação significativa negativa e baixa das variáveis movimento humano $(M)$ e proporção de movimento humano ativo e passivo (Ma:Mp) com a idade, e correlação significativa positiva e baixa com a escolaridade, o que revela que a criatividade, o raciocínio abstrato, a empatia e a tomada de decisões apresentam-se mais proeminentes nos adultos mais jovens, bem como nos indivíduos que possuem maior escolaridade. A correlação significativa positiva e baixa da proporção das respostas globais e de movimento humano 


\section{H. INTERACÃO EM LTPSICOLOGIA}

Jucelaine Bier Di Domenico Grazziotin e Silvana Alba Scortegagna
(W:M) com a idade indica que os mais jovens apresentaram uma tendência à produtividade $(W)$ aquém de suas reais capacidades (M). Diferentemente, à medida que a idade avança $\mathrm{e}$ os adultos se tornam mais velhos, há uma tendência a estabelecer e cumprir objetivos e metas mais ousadas. A correlação significativa positiva e baixa das respostas populares $(P)$ com a escolaridade mostra que indivíduos com maior nível escolar apresentaram uma propensão de melhor adequação social.

\section{DISCUSSÃO}

O objetivo principal deste estudo foi buscar evidências de validade convergente entre indicadores do processamento cognitivo do Zulliger com a idade e a escolaridade. Os dados representam achados interessantes e podem trazer incremento à qualidade das práticas avaliativas com o uso do teste. No que tange aos aspectos cognitivos, os participantes obtiveram resultados nos parâmetros normativos nas variáveis $R, W: M, M, P, M a-M p$. No geral, são indicadores de energia, motivação, criatividade, raciocínio abstrato e lógico, de adequação social e determinação (Exner \& Sendin, 1999; Villemor-Amaral \& Primi, 2012).

A presente pesquisa seguiu as instruções vigentes do teste de Zulliger (Villemor-Amaral \& Primi, 2012) com respostas não controladas e obteve a média do número de respostas e do desvio padrão com valores similares aos parâmetros normativos de não pacientes (Franco et al., 2012). Contudo, os 64 participantes do presente estudo denotaram um padrão mais controlado de respostas (mínimo de seis e máximo de 14 respostas) quando comparados aos 220 indivíduos da tabela normativa de não pacientes (mínimo de dois e máximo de 20 respostas). Este aspecto pode oferecer diferença na qualidade psicométrica e interpretativa dos resultados do presente estudo comparativamente ao estudo normativo, pois se aproxima de estudos recentes que demonstram que protocolos com menos de seis respostas no Zulliger podem ter sua confiabilidade reduzida (Villemor Amaral \& Cardoso, 2018). Protocolos muito curtos ou longos demais podem comprometer a qualidade das interpretações e desestabilizar alguns indicadores. Assim, estudos sugerem ampliar e controlar o número de respostas no método de Zulliger (Gonçalves et al. 2019; Villemor-Amaral et al., 2016).

Estes dados comparativos sinalizam possibilidades e limitações do instrumento, alertam sobre a necessidade de avanços nas pesquisas, de estudos normativos, mas também incrementam a relevância do Zulliger na avaliação de indivíduos sem histórico de doenças ou problemas incapacitantes, que se mantêm ativos em suas ocupações na medida em que confrontam dados sobre semelhanças e particularidades de comportamento de um grupo específico, de trabalhadores do comercio, não pacientes; e outro mais am- plo, da tabela normativa de não pacientes. A empatia e iniciativa nas tomadas de decisões também foram características observadas na amostra estudada e corrobora estudos prévios (Ferreira \& Villemor-Amaral, 2005; Grazziotin \& Scortegagna, 2016) e a amostra normativa (Franco et al.,2012), assim como a capacidade produtiva e ajustamento social mostraram-se semelhantes com os resultados dos estudos (Franco \& Villemor-Amaral, 2012; Grazziotin \& Scortegagna, 2016), o que demonstra coerência entre os achados com o uso do instrumento em uma população de não pacientes .

0 aumento significativo de XA\% e a diminuição de $X-\%$ em comparação aos parâmetros normativos de não pacientes informam que os indivíduos tendem a perceber a realidade de modo objetivo, com o mínimo de distorções subjetivas (Villemor-Amaral \& Primi, 2012). 0 aumento na variável XA\% e diminuição em $X-\%$ em trabalhadores do comércio, não pacientes, foi demonstrado em estudo anterior (Grazziotin \& Scortegagna, 2016a). Esses achados motivam a pensar que as características individuais, de idade e/ou educativas, podem suscitar diferenças na forma como as pessoas respondem aos estímulos de uma tarefa de desempenho. Tais resultados asseveram a utilidade do Zulliger na avaliação cognitiva, associada ao processo de identificação e tradução da informação, quando se faz necessário avaliar a presença ou não de problemas mediacionais.

As variáveis $\mathrm{W}$ e $\mathrm{DQ}+$ indicam capacidade de planejamento e percepções que envolvem esforço cognitivo mais elaborado e criativo bem como processos de abstração e síntese, e motivação pelo trabalho (Villemor-Amaral \& Primi, 2012). 0 aumento significativo destas variáveis no grupo estudado cogita a ideia de que tais indivíduos tendem a buscar soluções criativas para resolver problemas. Neste sentido, a literatura aponta que adultos jovens e de meia idade com boas condições de saúde e que exercem diariamente atividades ocupacionais não costumam apresentam prejuízos em suas capacidades cognitivas e produtivas (Agarwal et al., 2009; Meyer et al., 2015; Salthouse, 2012).

Além das questões da personalidade, as variáveis idade e escolaridade também podem influenciar as funções cognitivas. A produtividade, por exemplo, mostrou-se associada com educação, inteligência e adaptação (Meyer et al., 2011) e a educação está entre as variáveis demográficas de associação mais consistente com os escores do Rorschach de adultos, particularmente no domínio do processo cognitivo (Meyer et al., 2015).

Algumas variáveis do teste de Zulliger demonstraram associação somente com a idade, outras apenas com escolaridade e outras com ambos os fatores. A capacidade de reconhecer a realidade de modo convencional observado nas respostas P (Villemor-Amaral \& Primi, 2012) associou-se positivamente em indivíduos com maior escolaridade. Este 


\section{M." INTERACÃO EM HA PSICOLOGIA}

Jucelaine Bier Di Domenico Grazziotin e Silvana Alba Scortegagna achado corrobora parcialmente achados de Ames et al. (1973) que encontraram aumento nas respostas $P$ em pesquisa com indivíduos em idades mais avançadas e de alta escolaridade. A adequação social $(P)$ verificada no Zulliger mostrou compatibilidade com as características da amostra estudada, de pessoas que interagem com clientes, o que reforça o potencial do instrumento.

A empatia, a criatividade, o raciocínio abstrato, a iniciativa e tomada de decisões (M, Ma:Mp) demonstraram aumentar significativamente nos adultos com maior escolaridade e diminuir nos mais velhos. Estes dados confirmam a $\mathrm{H} 1 \mathrm{e}$ ratificam os achados de Meyer et al. (2015) que encontram associação significativa e positiva entre $\mathrm{M}$ e escolaridade. Os dados igualmente confirmam a $\mathrm{H} 2$ e corroboram Salthouse (2012) e Hartshorne e Germine (2015) que evidenciaram que adultos jovens costumam processar informações e tomar decisões com raciocínio lógico e maior rapidez, e adultos de meia idade podem apresentar maior acúmulo e capacidade de utilização do conhecimento e experiência adquiridos. Este dado é observado no presente estudo, já que os mais jovens, pouco experientes, são normalmente orientados e supervisionados por colegas mais velhos, mais experientes.

Meyer et al. (2015), não encontraram diferenças significativas do movimento humano $(\mathrm{M})$ em relação à idade. Os autores consideram que muitas das pesquisas que apresentavam diferença nos escores de adultos tinham alguns problemas metodológicos e nos critérios de inclusão e exclusão (difıculdades cognitivas e ausência de dificuldades cognitivas na mesma amostra) e, embora as evidências atuais na idade adulta não sejam conclusivas, as respostas dos adultos jovens e mais velhos não parecem diferir notavelmente. Portanto, o seguimento de estudos dessa natureza deve ser considerado em futuras pesquisas.

Ainda que, no presente estudo, o avanço da idade tenha acarretado diminuição significativa nas variáveis que representam aspectos dinâmicos e de tomadas de decisões (M, Ma:Mp), não ocorreram diferenças significativas naquelas que envolvem processos de pensamento, de percepção e adequação à realidade (XA\% e X-\%). Em conformidade com estes resultados, outros estudos não encontraram diferenças significativas nestas variáveis entre adultos jovens e mais velhos (Meyer et al., 2015), e o método de Zulliger evidenciou ajuste perceptivo da realidade, ausência de percepções inadequadas (X-\%) em idosos com cognição preservada e alto nível de escolaridade (Aguilar \& Graso, 2015). Estes achados podem ser derivados, em parte, das boas condições de saúde e do nível elevado de escolaridade que potencializam a reserva cognitiva em todas as idades (Meléndez et al., 2013; Zimmermann et al., 2014). Os dados parecem promissores ao revelarem que adultos saudáveis, jovens e mais velhos, não são propensos a fornecerem muitas respostas de forma distorcida.
A representação conceitual que a pessoa faz da realidade orienta suas ações no ambiente (Villemor-Amaral \& Primi, 2012), o julgamento adequado da realidade ajuda as pessoas a avaliarem as implicações das reações interpessoais com precisão e apreenderem os limites do comportamento adequado, em diferentes situações, e auxiliam a antecipar as consequências prováveis de suas ações e atingir metas (Exner \& Sendín, 1999). Neste sentido, a análise de desempenho em funções diversas revela que a produtividade, a quantidade de tarefas executadas e tomadas de decisões podem diminuir com o avanço da idade, mas a qualidade mantém-se constante na maior parte da vida adulta e frequentemente melhora a partir dos 20 anos até os 70 anos de idade (Agarwal et al., 2009; Salthouse, 2012).

A busca por metas e objetivos, aspectos relacionados à liderança e ambição intelectual (W:M) aumentaram à medida que a idade avançava, confirmando a H2. Este achado é outro dado relevante e concorda com ideias de autores (Agarwal et al., 2009; Salthouse, 2012) que consideram que, com o passar dos anos, pode ocorrer deslocamento da motivação e das responsabilidades e, então, as pessoas executam menos tarefas e assumem mais trabalhos voltados à supervisão e orientação. Assim, adultos de meia idade podem apresentar maior acúmulo e capacidade de utilização de conhecimentos adquiridos (Hartshorne \& Germine, 2015; Salthouse 2012).

Os processos cognitivos podem ser afetados pela idade e escolaridade, mas outros fatores também precisam ser examinados (Argimon et al., 2014; Zimmermann et al., 2014). Cuidados com a saúde, motivação, disposição para pensar de determinada maneira, experiências de vida e acúmulo de informações podem interferir e compensar eventuais perdas cognitivas em adultos mais velhos. Igualmente, a conjunção de escolaridade e atividades ocupacionais faz com que o indivíduo utilize mais redes de conexão cerebral que operam como fator de reserva e proteção contra o declínio cognitivo (Meléndez et al., 2013; Salthouse, 2012; Zimmermann et al., 2014).

Isso é particularmente importante no âmbito empresarial que considera a dinâmica do indivíduo e as competências necessárias para a execução das funções. A maior parte da população trabalhadora e economicamente ativa encontra-se na faixa de 18 a 49 anos e o contingente de adultos com 50 anos e mais tem aumentado nas organizações (PME, IBGE, 2016), impulsionando empresas a investir nos processos de seleção de pessoal, na busca de talentos e no bem-estar dos seus funcionários. Também importa para orientar as organizações na tomada de decisões e para atender à crescente preocupação com o desenvolvimento ético profissional e bem-estar dos membros que dela fazem parte (Widhiarso \& Himam, 2015). 


\section{INTERACCÃO EM PSICOLOGIA}

Para as empresas realizarem intervenções que auxiliem no desenvolvimento de seus funcionários, conhecer seus talentos, suas potencialidades e seus limites são necessárias avaliações periódicas. Assim, o uso de instrumentos sensíveis, capazes de examinar e trazer compreensões do funcionamento mental do indivíduo e de seu contexto demográfico (Meyer et al., 2015; Primi et al., 2012), pode permitir interpretações mais fidedignas dos achados e a tomada de decisões apropriadas. Avaliações psicológicas de qualidade e que considerem todas as questões éticas e sociais podem auxiliar populações de diversas idades e contextos (Conselho Federal de Psicologia - CFP, 2018).

Portanto, é importante destacar que as principais contribuições deste estudo revelam: a) evidências de validade convergente do teste de Zulliger com a idade e escolaridade; b) implicações das variáveis escolaridade e idade nos processos cognitivos e, consequentemente, no desempenho das funções e dos resultados nas empresas. Pode-se dizer que este estudo trouxe enriquecimento para a caracterização dos aspectos cognitivos de adultos.

Contudo, os dados obtidos precisam ser interpretados com cautela, observando como limitação os achados serem balizados em estudo transversal, com amostra restrita, o que não permite que possam ser generalizados para todas as pessoas e empresas. Como se pode observar, a falta de estudos em profundidade entre medidas de performance e a influência da idade e educação sobre o funcionamento cognitivo na vida adulta não permitem entendimento conclusivo (Meyer et al., 2015).

Pesquisas futuras devem realizar investigações com amostras mais extensas e de diferentes regiões, utilizando grupos comparativos contrastantes, não pacientes e pacientes, e funções diversificadas, com indivíduos de diferentes faixas etárias e em idades mais avançadas, tendo em vista a longevidade da população (Cardoso et al., 2018, Grazziotin \& Scortegagna, 2016b) e os dados vigentes sobre a população ativa no mercado de trabalho (IBGE, 2016). Além disto, considerando a amostra do presente estudo, adultos jovens e de meia idade, o objetivo desta pesquisa foi de explorar as variáveis $R, W, W: M, M, X A \%, X-\%, D Q+, P, M a-M p$, representativas de produtividade, empatia, percepção da realidade, adequação social e raciocínio abstrato, as quais poderiam por hipótese ser influenciadas por questões de idade e/ ou escolaridade. Estudos futuros longitudinais poderiam acrescentar outras variáveis da tríade cognitiva, como, por exemplo, MOR, Wsum6, S-, PSV, DQV, M-, que representam dificuldades e limitações nos processos cognitivos (Grazziotin \& Scortegagna, 2018) e podem ser indicadores de patologias. Outras variáveis não fizeram parte das hipóteses (WDA\%, Xu\%, S-), ainda que parcialmente contempladas $(\mathrm{XA} \%, \mathrm{~W}, \mathrm{X}+\%)$, poderão ser abordadas em estudos futuros.
Jucelaine Bier Di Domenico Grazziotin e Silvana Alba Scortegagna
Por fim, considerando-se a inexistência de estudos com o teste de Zulliger e a temática - processos cognitivos, idade e escolaridade -, estudos comparativos com e sem controle do número de respostas e testando outros arranjos de interpretação, como comparar grupos de diferentes idades e escolaridades, correlacionando essas variáveis com outros instrumentos, poderão melhor consolidar as propostas que cercam esta temática (Gonçalves et al., 2019; Villemor-Amaral \& Cardoso, 2018; Villemor-Amaral et al., 2016) e trazer incremento ao acúmulo de evidências de validade do teste de Zulliger.

\section{DECLARAÇÃO DA CONTRIBUIÇÃO DOS AUTORES}

Certificamos que todos os autores participaram suficientemente do trabalho para tomar pública sua responsabilidade pelo conteúdo. A contribuição de cada autor pode ser atribuída como se segue:

Jucelaine Bier Di Domenico Grazziotin: Responsável pela concepção e delineamento do estudo, análise e interpretação dos dados, redação crítica relevante do conteúdo do manuscrito, por todos os aspectos do trabalho, incluindo a garantia de sua precisão e integridade.

Silvana Alba Scortegagna: Participou da concepção e delineamento do estudo, análise e interpretação dos dados e redação crítica do conteúdo do manuscrito, responsável pela aprovação final da versão a ser publicada, incluindo a garantia de sua precisão e integridade.

\section{DECLARAÇÃO DE CONFLITOS DE INTERESSE}

Os autores declaram que não há conflitos de interesse no manuscrito submetido.

\section{REFERÊNCIAS}

Aguilar, M. J. G., \& Graso, L. (2015). Evaluación de una muestra de adultos mayores sanosde población general a través del test de Zulliger-Sistema Comprehensivo. Psicodiagnóstico de Rorschach, 36 (1), 45-71. http://www.asoc-argrorschach.com.ar/revista/Revista-Rorschach-dic-2015-Anio-36-N-1.pdf\#page=43

American Educational Research Association [AERA], American Psychological Association [APA], Nacional Council on Measurement in Education [NCME] (2014). Standards for Educational and Psychological Testing: American Educational Research Association.

Ames, L. B., Metraux, R. W., Rodell, J. L., \& Walker, R. N. (1973). Rorschach responses in old age (Rev. ed.). Oxford, England: Brunner/Mazel. 


\section{H MTERAC̄OOEM LF. PSICOLOGIA}

Agarwal, S.,Driscoll, J. C., Gabaix, X. \& Laibson, D. (2009). The age of reason: financial decisions over the lifecycle with implications for regulation. Brookings Papers on Economic Activity 2, 51-117. https://doi:10.2139/ssrn.973790

Argimon. I. L., Irigaray, T. Q., \& Stein, L. M. (2014). Cognitive development across different age ranges in late adulthood. Universitas Psychologica, 13(1), 253-264. doi: 10.11144/-Javeriana.UPSY13-1.cdad

Cardoso, L., Gomes, G., Pacheco, F., \& Viana, J. (2018). Análise da produção de artigos científicos brasileiros sobre o Teste de Zulliger. Interação em Psicologia, 22(3). doi: 10.5380/ psi.v22i3.45821

Cabeza, R., Albert, M., Belleville, S., Craik, F. I. M., Duarte, A., Grady, C. L., Rajah, M. N. (2018). Maintenance, reserve and compensation: the cognitive neuroscience of healthy ageing. Nature Reviews Neuroscience, 19, 701-710. https://doi:10.1038/s41583-018-0068-2

Conselho Nacional de Saúde (2016). Resolução No 510, de 07 de abril de 2016.http://conselho. saude.gov.br/ resolucoes/2016/reso510. pdf

Conselho Federal de Psicologia - CFP. (2018). Resolução CFP No 09/2008. http://satepsi.cfp.org.br/docs/Resolu\%C3\%A7\%C3\%A3oCFP-n\%C2\%BA-09-2018-com-anexo.pdf

Evans, F. B. \& Finn, S. E. (2016). Training and consultation in psychological assessment with professional psychologists: Suggestions for enhancing the profession and individual practices. Journal of Personality Assessment, 99(2), 175-185. https://doi:10.1080/00223891.2016.1187156

Exner, J.E. Jr. \& Sendín, C. (1999). Manual de Interpretação do Rorschach para o sistema Compreensivo: Casa do Psicólogo.

Ferreira, M. E. A., \& Villemor-Amaral, A. E. (2005). 0 teste de Zulliger e avaliação de desempenho. Paidéia 15(32), 367376. http://www.scielo.br/pdf/paideia/v15n32/06.pdf

Franco, R. R. C., \& Villemor-Amaral, A. E. (2012). Validade incremental do Zulliger e do Pfister no contexto de toxicomania. Psico-USF, 17(1), 73-83. https://doi:10.1590/ S1413-82712012000100009

Franco, R. R. C., Cardoso, L. M., Villemor-Amaral, A. E. \& Primi, R. (2012). Estudos de Normatização. In: Villemor-Amaral, A. E., Primi, R. (Orgs.). Teste de Zulliger no Sistema Compreensivo ZSC: Forma individual. (2ª Ed.). Casa do Psicólogo, 137-170.

Gonçalves, A. P., Zuanazzi, A. C., \& Villemor-Amaral, A. E. (2019). Aplicação R-Otimizada no Zulliger: evidências de validade com pacientes depressivos. Avaliação Psicológica, 18(2), 111-120. https://dx.doi.org/10.15689/ ap.2019.1802.16215.01

Grazziotin, J. B. D., \& Scortegagna, S. A. (2016a). Mediação no Zulliger: Evidências de Validade em Amostra de Não Pacientes. Psico-USF, 21(1), 63-7. https://doi:10.1590/141382712016210106
Jucelaine Bier Di Domenico Grazziotin e Silvana Alba Scortegagna
Grazziotin, J. B. D., \& Scortegagna, S. A. (2016b). Revisão de pesquisas brasileiras sobre o Teste de Zulliger publicadas em artigos. Avaliação Psicológica, 15(2), 227-235. https:// doi: 10.15689/ap.2016.1502.11

Hartshorne J. K., \& Germine L.T. (2015). When does cognitive functioning peak? The asynchronous rise and fall of different cognitive abilities across the life span. Psychological science, 26(4), 433-43. https://doi. org/10.1177/0956797614567339

Instituto Brasileiro de Geografia e Estatística - IBGE. (2016). Pesquisa Mensal de Emprego - Fevereiro de 2016. https://www.ibge.gov.br/estatisticas/sociais/trabalho/ 9180-pesquisa-mensal-de-emprego.html?edicao $=9182$

Landis, J. R., \& Koch, G.G (1977). The measurement of observer agreement for categorical data. Biometrics, 33(1), 159-174.

Meléndez, J. C., Mayordomo, T., \& Sales, A. (2013). Comparación entre ancianos sanos con alta y baja reserva cognitiva y ancianos con deterioro cognitivo. Universitas Psychologica, 12(1), 73-80. https://core.ac.uk/download/ pdf/26197263.pdf

Meyer, G.J. (2017). What Rorschach performance can add to assessing and understanding personality. International Journal of Personality Psychology, 3(1), 36 - 49. https:// goo.gl/QHqg1C

Meyer, G. J., Viglione, D. J., Mihura, J. L., Erard, R. E., \& Erdberg, P. (2011). Rorschach performance assessment system: Administration, coding, interpretation and technical manual.: Rorschach Performance Assessment System.

Meyer, G. J., Giromini, L., Viglione, D. J., Reese, J. B., \& Mihura, J. L. (2015). The Association of Gender, Ethnicity, Age, and Education With Rorschach Scores. Assessment, 22(1) 46 64. https://doi: 10. 1177/10 73191114544358

Ministério do Trabalho (2018). Normas regulamentadora $n^{\circ}$ 07 - Programa de controle médico de saúde ocupacional [PCMSO]. Portaria n. ${ }^{\circ}$ 1.031/2018 de 06 de dezembro: Diário Oficial da União, https://enit.trabalho.gov.br/portal/ images/Arquivos_SST/SST_NR/NR-07.pdf

Pires, A. A. (2000). National norms for the Rorschach Comprehensive System in Portugal. In R. Dana (Ed.), Handbook of cross-cultural and multicultural personality assessment (pp. 367-392). Mahwah, NJ: Lawrence Erlbaum.

Primi, R., Muniz, M. E., \& Villemor-Amaral. A.E. (2012). Validade do Zulliger no Sistema Compreensivo. In: Villemor-Amaral, A. E., Primi, R. (Orgs.). Teste de Zulliger no Sistema Compreensivo ZSC: Forma individual. (2ª Ed.). Casa do Psicólogo, 137-170.

Salthouse, T. A. (2012). Consequences of age-related cognitive declines. AnnualReview of Psychology, 63(20), 1- 226. https://doi:10.1146/annurev-psych-120710 100328 
Viglione, D. J., Meyer, G., Jordan, R. J., Converse, G. L., Evans, J., MacDermott, D., \& Moore, R. (2015). Developing an Alternative Rorschach Administration Method to Optimize the Number of Responses and Enhance Clinical Inferences. Clinical Psychology \& Psychotherapy, 22(6), 546-558. https://doi:10.1002/cpp.1913

Villemor-Amaral, A. E., \& Primi, R. (2012). Teste de Zulliger no Sistema Compreensivo ZSC: Forma individual. ( $2^{\mathrm{a}} \mathrm{Ed}$.). Casa do Psicólogo.

Villemor Amaral, A.E. \& Cardoso, L. M. (2018). Atualizações sobre o Zulliger. Em: C. S.Hutz; D. R., Bandeira \& C. M.Trentini (Orgs.). Avaliação psicológica da inteligência e da personalidade (pp. 354-365). Artmed.
Villemor-Amaral, A. E., Pianowski, G., \& Carvalho, L, F. (2016). Issues about color, human movement and number of responses in the Zulliger test. Rorschachiana: Journal of the international society for the Rorschach. 37(2), 95-113. https://doi:10.1027/1192-5604/a000068

Widhiarso, W. \& Himam, F. (2015). Employee Recruitment: Identifying Response Distortion on the Personality Measure. EJBO - Electronic Journal of Business Ethics and Organization Studies, 20 (1), 14-21. http://ejbo.jyu.fi

Zimmermann, N., Parente, M.A. M. P., Joanette, Y., \& Fonseca, R. P. (2014). Unconstrained, Phonemic and Semantic Verbal Fluency: Age and Education Effects, Norms and Discrepancies. Psicologia: Reflexão e Crítica, 27(1), 55-63. https://doi:10.1590/S0102-79722014000100007 